\title{
A Simple Iterative Algorithm to achieve utility proportional fairness
}

\author{
Ye Ying-ze \\ Modern Education Technology Center \\ Huazhong Agricultural University \\ Wuhan, China \\ walking7876@sina.com
}

\author{
Zhang Ying \\ Department of Computer Science, College of Science \\ Huazhong Agricultural University \\ Wuhan, China \\ zy@mail.hzau.edu.cn
}

\begin{abstract}
Paper is concerned with the distributed algorithm of utility proportionally fair bandwidth allocation. We propose a converges faster algorithm to achieve utility proportional fairness. The simulation results verify the performance.
\end{abstract}

\section{Keywords-Resource allocation; Utility proportional fairness}

\section{INTRODUCTION}

Allocating bandwidth fairly is a key issue in computer networks. Given the link capacities of a network and a set of traffic flows, the task is to determine the transmission rates of the flows so that given fairness criteria are meet. The most common fairness criterion is bandwidth max-min fairness, in which the lowest transmission rate that a flow achieves is maximized [1]. This criterion gives an absolute priority to the smaller flows, which leads to low total flow rates. Proportionally fair allocation [2] maximizes the sum of logarithms of the flow rates. It can be achieved by distributed primal-dual algorithms to solve a new systematic model called "Optimal Flow Control" (OFC) proposed by Kelly [3]. In this model, the objective is to maximize the aggregate source utility over their transmission rates. Based on this model, Low [4] proposed a algorithm through simply solving the dual of OFC model using gradient projection algorithm. J.Mo [5] define a family of fair allocations as outcomes of a utility maximization problem. A parameterized utility function is assumed to be common for all the flows. By changing the parameter of the utility function, different fair allocations emerge when the sum of user utilities is maximized. These allocations include max-min fairness, proportional fairness and the rate vector maximizing the end-to-end throughput.

Even though considerable progress has been made in this direction, the existing work focuses only on elastic traffic, because they all assumed the user utility to be concave. Another drawback of these traditional fairness criteria is that they implicitly require the users having the same utility function. In practice, different flows have very different bandwidth requirements, so different utility functions. A user running an application does not care about any fair bandwidth shares, as long as his application performs satisfactory. Hence, an application performance measures the utility that should be shared fairly among flows instead of bandwidth.
In [6], Cao and Zegura present a link algorithm that achieves a utility max-min fair bandwidth allocation, where for each link the utility functions of all flows sharing that link is maintained. In [7], Cho and Song present a utility max-min architecture, where each link communicates a supported utility value to sources using that link. Then sources adapt their sending rates according to the minimum of these utility values. In [8], the authors extend the utility max-min fairness and propose a new fairness criterion, utility proportional fairness, which includes the utility max-min fair resource allocation as a special case. A utility proportional fair bandwidth allocation is characterized by the solution of an associated optimization. The benefit a user $s$ gains when sending at rate $x_{s}$ is evaluated by a new redefined utility $\mu_{s}\left(x_{s}\right)$ and the objective is to maximize aggregate $\mu_{s}\left(x_{s}\right)$ subject to capacity constraints. This criterion only assumes that the bandwidth utilities are monotonic increasing in a given interval. This is a natural assumption since any application will profit from receiving more bandwidth in a certain bandwidth interval. Furthermore, the authors propose a new distributed flow control algorithm which does not need any per-flow information at the links to achieve this fairness criterion.

Recently, Penttinen [9] proposed a simple-to-implement iterative scheme for determining the proportionally fair and, more generally, $\alpha$-fair ( $\alpha>1 / 2$ ) [5] flow rate allocations. In this paper we describe how the optimality conditions of the utility proportionally fair bandwidth allocation problem directly reduce to an extremely simple-to-implement iteration which can be used to compute the rate vectors corresponding to utility proportional fairness. We show that this method only needs the link price to update until the optimal point, then the optimal rate vectors can be directly achieved.

The rest of the paper is organized as follows. In Section II, we review the background of flow control problem and examine the recently proposed optimal flow control algorithm to achieve the utility proportional fairness. In Section III, we use the direct method to develop a new distributed algorithm which achieves the same fairness. In Section IV, we use simulation to verify the new algorithm and compare it with the algorithm in [8]. Finally, we conclude the paper in Section V. 


\section{THE UTILITY PROPORTIONALLY FAIR BANDWIDTH ALLOCATION PROBLEM}

Consider a network that consists of a set $\zeta=\{1,2, \ldots, L\}$ of unidirectional links with a capacity $c_{l}, l \in \zeta$. The network is shared by a set $S=\{1,2, \ldots S\}$ of sources. Each source $s$ is characterized by five parameters $\left\{\zeta_{s}, x_{s}, m_{s}, M_{s}, U_{s}\left(x_{s}\right)\right\}$. The path $\zeta_{s} \subseteq \zeta$ is a subset of links that connect each source $s$ to its destination, $x_{s}$ is the transmission rate satisfying $0 \leq m_{s} \leq x_{s} \leq M_{s}<\infty$, when $m_{s}$ and $M_{s}$ are the minimum and maximum transmission rates required by source $s$, respectively. $U_{s}\left(x_{s}\right): R^{+} \rightarrow R$ is a continuously increasing and bounded utility function which can be used as a QoS performance indicator for source $s$. Let $x=\left[x_{1}, x_{2}, \ldots x_{s}\right]^{T}$. For each link $l$, define $S_{l}=\left\{s \in S, l \in \zeta_{s}\right\}$, which is the set of sources that access $\operatorname{link} l$. Note that, $l \in \zeta_{s}$ if and only if $s \in S_{l}$.

Redefined user utility function

$\mu_{s}\left(x_{s}\right)=\int_{m_{s}}^{x_{s}} \frac{1}{U_{s}(y)} d y, \quad m_{s} \leq x_{s} \leq M_{s}$

Then the traditional OFC problem can be reformulated as the following optimization problem:

$$
\begin{aligned}
& \max _{m_{s} \leq x_{s} \leq M_{s}} \quad \mu(x)=\sum_{s=1}^{S} \mu_{s}\left(x_{s}\right) \\
& \text { Subject to } \quad \sum_{s \in S_{l}} x_{s} \leq c_{l}, \quad l=1, \ldots, L
\end{aligned}
$$

The original utility function $U_{s}\left(x_{s}\right)$ is non-negative, continuous and strictly increasing over the range $x_{s} \in\left[m_{s}, M_{s}\right]$. Therefore, $\mu_{s}\left(x_{s}\right)$ must be increasing and strictly concave.

The following distributed dual algorithm solves this optimization problem:

At time $t+1$, each link $l$ updates its link price $p_{l}$ according to

$$
p_{l}(t+1)=\left[p_{l}(t)+\gamma\left(x^{l}(t)-c_{l}\right)\right]^{+}
$$

Where $\gamma>0$ is a small step size, and $x^{l}(t)=\sum_{s \in S_{l}} x_{s}(t)$ is the aggregate source rate at link $l$. It says that if the aggregate source rate at link $l$ exceeds the link capacity $c_{l}$, the link price will be increased; otherwise it will be decreased. The projection $[z]^{+}=\max \{0, z\}$ ensures that the link price is always non-negative.

When each source $s$ receives feedback congestion information, i.e., the path price, which is the sum of the link prices along its path, it adopts the following source algorithm to update the source rate:

$$
\begin{aligned}
& x_{s}(t+1)= {\left[x_{s}(t)+\gamma\left(\frac{1}{U_{s}\left(x_{s}(t)\right)}-p^{s}(t)\right)\right]_{m_{s}}^{M_{s}} } \\
& \text { Where } \\
& p^{s}(t)=\sum_{l \in \zeta_{s}} p_{l}(t)
\end{aligned}
$$

is the path price of source $s$, $[z]_{a}^{b}=\max \{a, \min \{b, z\}\}$. According to the definition of utility function, it is clear that $x_{s}\left(p^{s}\right)$ given in (5) is decreasing over the path price $p^{s}$.

Definition 1: A bandwidth allocation $x^{*}=\left[x_{1}{ }^{*}, x_{2}{ }^{*}, \ldots x_{s}{ }^{*}\right]^{T}$ is utility proportional fair, if it is feasible and for any other feasible allocation $x$,

$$
\sum_{s \in S} \frac{x_{s}-x_{s}^{*}}{U_{s}\left(x_{s}^{*}\right)}<0
$$

When the flow control algorithm (4) and (5) converges to the equilibrium $\left(x^{*}, p^{*}\right)$, the objective function (2) is maximized within the feasible solution. For all feasible allocation $x \neq x^{*}$, the optimality condition is

$$
\sum_{s \in S} \frac{\partial \mu_{s}\left(x_{s}^{*}\right)}{\partial x_{s}}\left(x_{s}-x_{s}^{*}\right)=\sum_{s \in S} \frac{x_{s}-x_{s}^{*}}{U_{s}\left(x_{s}^{*}\right)}<0
$$

Where the strict inequality follows from the strict concavity of $\mu_{s}\left(x_{s}\right)$.According to Definition 1, it is clear that, at optimality, the resource allocation $x^{*}$ is utility proportional fairness.

\section{THE NEW ALGORITHM TO ACHIEVE UTILITY PROPORTIONAL FAIRNESS}

Note that since both the objective function (2) and the constraint conditions (3) are convex, the optimum solution of the problem is characterized by the KKT conditions [10]. These can be formulated as

$$
\begin{gathered}
\frac{1}{U_{s}\left(x_{s}\right)}-\sum_{l \in \zeta_{s}} p_{l}=0, \quad \forall s \in S \\
p_{l}\left(c_{l}-\sum_{s \in S_{l}} x_{s}\right)=0, \quad \forall l \in \zeta \\
\sum_{s \in S_{l}} x_{s} \leq c_{l}, \quad \forall l \in \zeta \\
p_{l} \geq 0, \quad x_{s} \geq 0, \forall l \in \zeta, \forall s \in S
\end{gathered}
$$


Where the variables $p_{l}$ are the Lagrangian multipliers associated to the problem.

The conditions (9) give us

$$
x_{s}=U_{s}^{-1}\left(\frac{1}{\sum_{l \in \zeta_{s}} p_{l}}\right), \forall s \in S
$$

Using (13) to replace $x_{s}$ in conditions (10) we get

$$
p_{l}=p_{l} \sum_{s \in S_{l}} U_{s}^{-1}\left(\frac{1}{\sum_{l \in \zeta_{s}} p_{l}}\right) / c_{l}, \quad \forall l \in \zeta
$$

This provides us a convenient way of solving the problem with the following iteration:

$$
p_{l}(t+1)=p_{l}(t) \sum_{s \in S_{l}} U_{s}^{-1}\left(\frac{1}{\sum_{l \in \zeta_{s}} p_{l}(t)}\right) / c_{l}, \quad \forall l \in \zeta
$$

The iteration can be used to solve the problem (2), (3) starting from any initial values $p_{l}(0)>0$. When we get the optimal $p_{l}$, we can use condition to get the optimal $x_{s}$. The iteration has also the following interpretation. Consider the constraints (3) as in the demand-supply model. Note that at each iteration round the current price of link $l, p_{l}$, is multiplied by the demand to supply ratio of the resource $l$. If demand is larger than supply the price $p_{l}$ increases. Corresponding, the increased price decreases the demand on the next round.

Given that the iteration converges the limiting vector is optimal. Starting from a positive $p_{l}(0)$ the limiting $p_{l}{ }^{*}$ are clearly non-negative and thus both non-negativity constraints (12) hold. Furthermore, the iteration (15) is only a restatement of conditions (10) (13) meaning that both (9) and (10) hold at the converged solution. Finally, it is easy to see that at the converged solution the coefficient of $p_{l}(t)$ in (15), $\sum_{s \in S_{l}} x_{s} / c_{l}$, cannot be larger than 1 for any $l$, meaning that (11) holds. Thus the limiting vector $p^{*}$ together with the corresponding rates (13), satisfy the KKT conditions (9-12) and provide an optimal solution to the problem (2), (3).

\section{SIMULATION}

We use the same network in [13] to simplify the comparison. Consider the following simple network, as show in Fig.1, which consists of two links L1 and L2 with a capacity of $10 \mathrm{Mb} / \mathrm{s}$ and shared by three sources S1, S2 and S3. S1 traverses link L1 and L2, and S2 and S3 traverse L1 and L2, respectively. Their utilities are $U_{1}\left(x_{1}\right)=1 /\left(1+e^{-2\left(x_{1}-5\right)}\right), U 2\left(x_{2}\right)=0.125 x_{2}$, and $U 3\left(x_{3}\right)=\log \left(x_{3}+1\right) / \log 11$. The three sources have their maximum rate requirement set at 10,8 , and $10 \mathrm{Mb} / \mathrm{s}$, respectively.

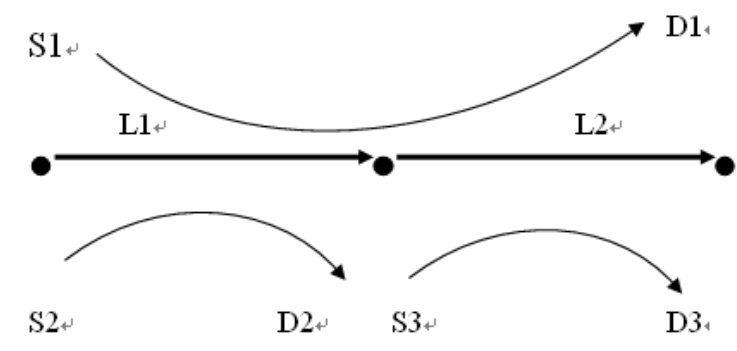

Fig.1. Network Topology with two links and three sources.

The starting point is an arbitrary positive vector, we set $p(0)=(4,4)^{T}$. Figure 2 shows how the values of $p_{1}$ and $p_{2}$ evolve step-by-step in the iteration (15).

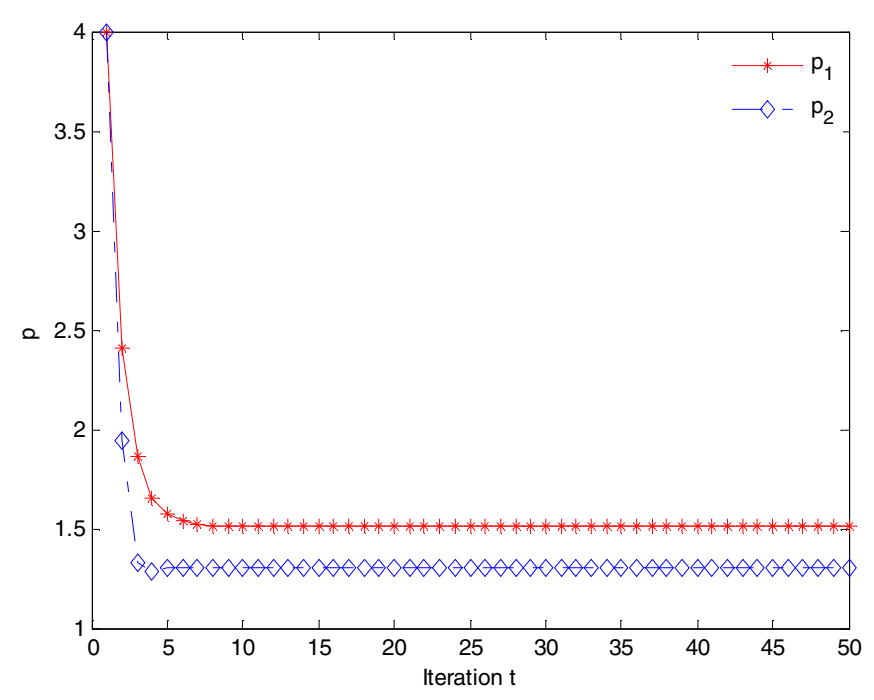

Fig.2. Values of $p_{1}$ (stars) and $p_{2}$ (diamond) in the iteration.

The iteration converges to $p^{*}=(1.5101,1.3031)$. Substituting this to (13) we get the utility proportionally fair allocation; 4.7052 Mbps, 5.2977 Mbps, 5.2977Mbps, for three sources respectively.

Figure 3 gives the link prices iteration by using algorithm (4) (5) for comparing. 
show that the algorithm converges faster than the algorithm

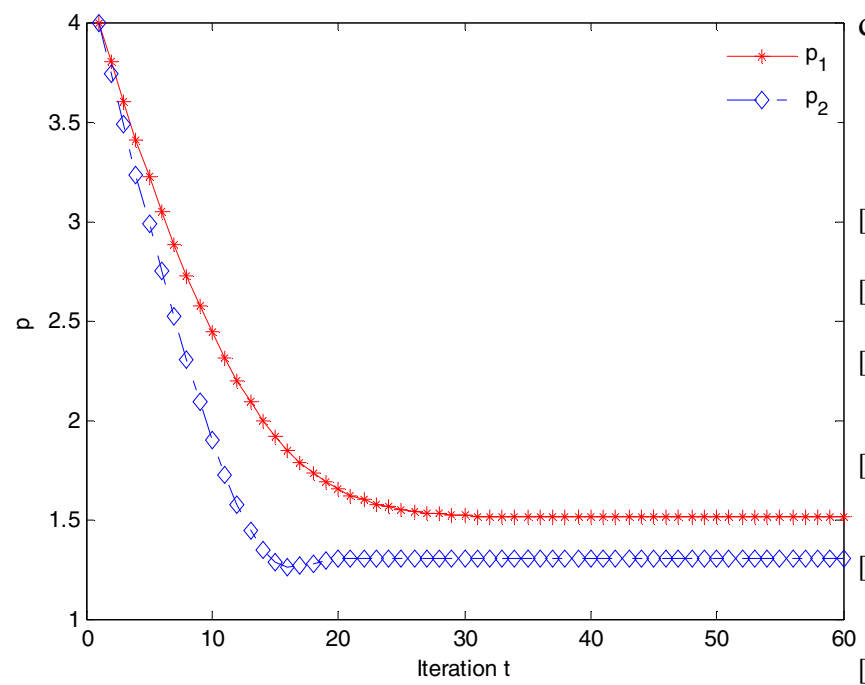
developed before.

\section{REFERENCES}

[1] D. Bertsekas and R. Gallager, Data Networks. Prentice-Hall, second edition, 1992

[2] Frank P. Kelly, "Charging and rate control for elastic traffic," European Trans. Telecommun., vol. 8, pp. 33-37, 1997.

3] F. P. Kelly, A. K. Maulloo, and D. K. H. Tan, "Rate control in communication networks: shadow prices, proportional fairness and stability," J. Operational Research Society, vol. 49, pp. 237-252, 1998.

[4] S. H. Low and D. E. Lapsley, "Optimization flow control, I: basic algorithm and convergence," "IEEE/ACM Trans. etworking, vol. 7, no. 6, pp. 861-874, Dec. 1999.

5] J. Mo and J. Walrand, "Fair end-to-end window-based congestion control," IEEE/ACM Trans. Networking, vol. 8. no. 5, pp. 556-567, Oct. 2000 .

[6] Cao, Z., Zegura, E. "Utility max-min: An application-oriented bandwidth allocation scheme”. In: Proceedings of- IEEE INFOCOM 99. (1999) 793-801.

Fig.2. Values of $p_{1}$ (stars) and $p_{2}$ (diamond) in the iteration by using the algorithm (4), (5).

Obviously, the iteration (15) converges much faster than (4). So we can use (5) and (15) to develop a new distributed source-link algorithm achieving utility proportional fairness bandwidth allocation.

\section{CONCLUSION}

We have proposed a new algorithm to solve utility proportional fairness bandwidth allocation. Simulation results

[7] J. Cho, S. Chong, "Utility max-min flow control using slope-restricted utility functions", To appear in Proceeding of GLOBECOM 2005

[8] Wei-Hua Wang, Marimuthu Palaniswami and Steven H. Low, "Application-Oriented Flow Control: Fundamentals, Algorithms and Fairness", IEEE/ACM Transactions on Networking (TON), vol. 14, No. 6, December 2006.

[9] Aleksi Penttinen, "Computation of Fair Flow Rates in Data Networks", IEEE Communications Letters, Vol 12, Issue 1, January 2008 Page(s):53 - 55

[10] M. Bazaraa, H. Sherali, and C. M. Shetty, Nonlinear Programming: Theory and Algorithms. Wiley, second edition, 1993. 